\title{
Hepatic Pathology among Patients without Known Liver Disease Undergoing Bariatric Surgery: Observations and a Perspective from the Longitudinal Assessment of Bariatric Surgery (LABS) Study
}

David E. Kleiner, MD, PhD ${ }^{1}$ Paul D. Berk, MD ${ }^{2}$ Jesse Y. Hsu, PhD ${ }^{3}$ Anita P. Courcoulas, MD, MPH David Flum, MD, MPH ${ }^{5}$ Saurabh Khandelwal, MD $^{5}$ John Pender, MD ${ }^{6}$ Alfons Pomp, MD ${ }^{7}$ James Roerig, PharmD ${ }^{8}$ Laura L. Machado, MD ${ }^{9}$ Bruce M. Wolfe, MD ${ }^{10}$ Steven H. Belle, PhD, MScHyg ${ }^{3}$ for the LABS Consortium

${ }^{1}$ Laboratory of Pathology, National Cancer Institute, Bethesda, Maryland

2 Department of Medicine, Columbia University, College of Physicians Address for correspondence David E. Kleiner, MD, PhD, Laboratory of Pathology/NCl, Bldg 10, Room 2B50, MSC 1500, 10 Center Dr., and Surgeons, New York, New York

${ }^{3}$ University of Pittsburgh Graduate School of Public Health, Pittsburgh, Pennsylvania

${ }^{4}$ Department of Surgery, University of Pittsburgh Medical Center, Pittsburgh, Pennsylvania

${ }^{5}$ Department of Surgery, University of Washington, Seattle, Washington

${ }^{6}$ Department of Surgery, East Carolina University, Greenville, North Carolina

${ }^{7}$ Department of Surgery, Weill Cornell Medical College, New York, New York

8 Neuropsychiatric Research Institute, University of North Dakota, Fargo, North Dakota

${ }^{9}$ Sacramento Bariatric Medical Associates, Sacramento, California

${ }^{10}$ Division of General Surgery, Oregon Health and Science University, Oregon Weight Loss Surgery, Portland, Oregon Bethesda, MD 20892 (e-mail: kleinerd@mail.nih.gov).

Semin Liver Dis 2014;34:98-107.

\section{Abstract \\ Keywords \\ - bariatric surgery \\ - liver biopsy \\ - NAFLD \\ - steatohepatitis \\ - cirrhosis}

Liver biopsy is not routine during bariatric surgery. Alanine aminotransferase (ALT) is widely used to screen for liver disease. We assessed the relationship between ALT and pathology in biopsies from Longitudinal Assessment of Bariatric Surgery (LABS) patients with normal preoperative ALTs. Biopsies from the LABS-1 and LABS-2 studies were scored using the NASH CRN and Ishak systems. Diagnosis and histology were examined in relation to alanine aminotransferase (ALT) values. Six-hundred ninety-three suitable biopsies were evaluated. Biopsied patients had a median age of 45 years; $78.6 \%$ were female and $35.1 \%$ diabetic; median body mass index was $46 \mathrm{~kg} / \mathrm{m}^{2}$. Six-hundred thirtyfive biopsied patients had preoperative ALTs. Median ALT was $25 \mathrm{IU} / \mathrm{L}$ (interquartile range [IQR] 19-36 IU/L); 26.6\% had an ALT > $35 \mathrm{IU} / \mathrm{L}$ and $29.9 \%$ exceeded the more restrictive Prati criteria for normal. Using the Prati criteria, $7.9 \%$ of participants with normal ALT had steatohepatitis and 5.3\% had $\geq$ stage 2 fibrosis. Logistic regression models were used to predict the probabilities of having bridging fibrosis/cirrhosis or a 
diagnosis of borderline/definite steatohepatitis in the unbiopsied LABS-2 sample. The proportion of biopsied participants with these findings was very similar to the modeled results from the unbiopsied cohorts. We estimated that $86.0 \%$ of participants with advanced fibrosis and $88.1 \%$ of participants with borderline/definite steatohepatitis were not biopsied and went undiagnosed. As ALT did not reliably exclude significant obesity-related liver disease in bariatric surgery patients, consideration should be given to routine liver biopsy during bariatric surgery and medical follow-up of significant hepatic pathology.

Obesity is the most significant public health issue currently facing the U.S. population, of whom $\sim 33 \%$ are overweight (body mass index [BMI] 25-30) and 36\% are obese (BMI $>30) \cdot{ }^{1-4}$ It is the predominant cause of nonalcoholic fatty liver disease (NAFLD) in the United States and Western Europe, ${ }^{5,6}$ although not necessarily in other parts of the world, ${ }^{7}$ and is also associated with a variety of other serious consequences. ${ }^{8}$ NAFLD encompasses a spectrum from hepatic steatosis through nonalcoholic steatohepatitis (NASH) to cirrhosis and end stage liver disease. ${ }^{9-12}$

Although steatosis and its association with obesity, and steatohepatitis and its association with alcohol abuse have long been known, the first, underappreciated descriptions of nonalcoholic steatohepatitis appeared in $1952^{13}$ and $1971 .^{14}$ The designation nonalcoholic steatohepatitis and its acronym NASH were reported by Ludwig et al in $1980,{ }^{15}$ and in a seminal series by Powell et al in $1990 .{ }^{16}$ While initially considered a rare entity, the prevalence of NASH, along with NAFLD, has increased dramatically with that of obesity. As the epidemic of new cases of hepatitis $C$ wanes $^{17,18}$ and new antiviral therapies improve the treatment of chronic hepatitis $\mathrm{C}$ infection, ${ }^{19-22}$ end-stage liver disease evolving from NAFLD via NASH is projected to become the major indication for liver transplantation, perhaps as early as $2025 .^{23-25}$

There is no generally accepted pharmacologic treatment for NAFLD despite some positive trials (e.g., ${ }^{26}$ ). The failure to obtain consistent responses to proposed therapies may reflect the growing diversity of genotypes in which a phenotypically similar NAFLD has been detected by means of Genome-Wide Association Scan (GWAS) studies. ${ }^{27}$ Bariatric surgery offers an alternative, and some would argue the best, option for the treatment of obesity, which is widely presumed to be the basis for most cases of NAFLD.

The LABS consortium was created to assess the long-term durability of weight loss and the associated benefits to other health outcomes following bariatric surgery. ${ }^{28}$ When the major LABS protocols were initiated nearly a decade ago, much of the focus was on the potential impact on diabetes and associated cardiovascular disease. With the increasing recognition of the role of obesity in the pathogenesis of serious liver disease in Western countries, the incidence of the various stages of NAFLD in bariatric surgery patients, whether they could be identified by noninvasive methods, and the impact of bariatric surgery on liver disease outcomes have become more critical issues. This report summarizes the histologic findings in 693 liver biopsies, obtained during the course of 6,382 primary bariatric procedures performed as part of the LABS- 1 and LABS- 2 studies conducted by the NIDDK-funded Longitudinal Assessment of Bariatric Surgery (LABS) consortium. Biopsy results were analyzed with respect to the patients' ALT results, assessed against two different values for the upper limit of normal (ULN), the LABS consensus criterion (ULN $<35 \mathrm{IU} / \mathrm{L}$ ) and by the stricter criteria of Prati et $\mathrm{al}^{29}$ (Men: ULN < $30 \mathrm{IU} / \mathrm{L}$, Women: ULN < $19 \mathrm{IU} / \mathrm{L}$ ), and were also evaluated according to the frequency with which the patient's surgeon performed liver biopsies during bariatric operations.

\section{Methods}

\section{Participants}

The biopsies reviewed in this report were obtained from the 6,383 patients who underwent primary bariatric surgical procedures in either the LABS- 1 or LABS- 2 study. The general design of these studies and procedures for patient recruitment and consent were described earlier. ${ }^{30}$ LABS- 1 was a short-term study that focused on significant complications including mortality in the 30 days after an initial bariatric surgical procedure. As part of LABS-1, 4,776 patients underwent a bariatric surgical operation. The characteristics of the patient cohort and the results of LABS-1 have already been reported. ${ }^{31}$ The LABS-2 study, which is currently in its seventh year, involves more detailed, longer-term follow-up of patients after bariatric surgery. The LABS-2 cohort is comprised of 2,458 patients. The composition of the cohort and 3-year outcomes have recently been reported. ${ }^{32}$

\section{Biopsies}

Liver biopsies were obtained during bariatric surgeries according to the local standard-of-care and using local standards for biopsy technique. Participants enrolled in LABS-2 were consented for liver biopsy review by the study pathologist at the time of enrollment. Consent for biopsy review was obtained from LABS-1 participants retrospectively. Hematoxylin and eosin and Masson trichrome stains were available on all biopsies. The study pathologist (DEK) was blinded to all clinical and demographic data. Biopsy adequacy was determined by measuring the length of the biopsy in $\mathrm{mm}$ and by counting portal areas. Biopsies measuring $10 \mathrm{~mm}$ in length or 
having 10 portal areas were generally considered adequate for scoring, while smaller biopsies that nevertheless still could be scored were considered suboptimal. Biopsies graded as inadequate were not scored. Features of fatty liver disease were scored using the system devised by the NASH Clinical Research Network (NASH CRN). ${ }^{12}$ The NAFLD Activity Score (NAS) was used as a composite measure of injury. Inflammation and fibrosis were further evaluated using the Ishak scoring system. ${ }^{33}$ In addition to scoring the biopsies, each biopsy was categorized into one of four histological patterns: "Not Steatohepatitis," "Borderline, Zone 3 Pattern," "Borderline, Zone 1 Pattern" and "Definite Steatohepatitis," according to definitions used by the NASH CRN. ${ }^{11}$

\section{Statistical Analysis}

Sample medians and interquartile ranges (IQRs) are shown for continuous variables, and the Wilcoxon sum-rank test is used for testing for equality of distributions. Nominal level variable distributions are shown as percentages and tested for equality using Pearson's chi-square or Fisher exact test. For ordinal variables, the Cochran-Armitage test for trend is used. Two multiple logistic regression models are fit to estimate the probability of having significant fibrosis and having the diagnosis of borderline or definite steatohepatitis, adjusting for key covariates. Statistical analyses are performed using SAS 9.2 (SAS Institute Inc., Cary, NC).

\section{Results}

Twenty surgeons from eight clinical centers contributed biopsies from the LABS- 1 and 2 cohorts that were obtained as part of routine clinical care at their respective centers. Three-hundred sixty-three biopsies were obtained from the
2,458 first surgeries in LABS-2. No biopsy was performed during 2,095 of these surgeries. Of the 363 biopsies obtained, 47 were not forwarded for review by the local pathologist, 6 patients declined consent, and slides from 1 biopsy were lost in transit. Thus, slides from 309 biopsies were submitted for review. This number was substantially augmented by biopsies performed during the LABS-1 study at a single center, at which the standard of care was to biopsy all patients during bariatric surgery. Four-hundred forty-one biopsies were performed during the 475 surgeries at this center. At the time of this review, slides from 22 of the biopsies could not be retrieved; slides from the remaining 419 were sent for review.

Of the 728 biopsies available for review, 35 (4.8\%) were deemed inadequate for scoring, mainly due to the size and quality of the sample. The remaining 693 scored biopsies were taken from patients with a median age of 45 years (IQR 36 to 55 years); $78.6 \%$ were female. Their median BMI was 46 $\mathrm{kg} / \mathrm{m}^{2}$ (IQR 42 to $52 \mathrm{~kg} / \mathrm{m}^{2}$ ) and $35.1 \%$ were diabetic. Most of the participants (87.7\%) self-identified as Caucasian. By comparison, the median age of the 6,382 patients who comprised the total LABS surgical cohort was 45 years (IQR 36-54 years); $78.9 \%$ were female; their median BMI was $46 \mathrm{~kg} / \mathrm{m}^{2}$ (IQR 42 to $52 \mathrm{~kg} / \mathrm{m}^{2}$ ); 33\% were diabetic (-Table 1).

Because biopsies were only obtained as part of the locally established standard of care, there was a wide variation with respect to the proportion of biopsies performed among the total number of bariatric procedures. Of the 38 participating surgeons, 3 had a high biopsy rate $(89.6 \%, 92.7 \%$ and $94.1 \%$ of first surgeries), 8 had biopsy rates between 20 and $80 \%, 20$ had biopsy rates less than $20 \%$, and 7 surgeons performed no biopsies. To gain perspective on the differences in the various samples, data from participants ( - Table 1) and biopsies (-Table 2) for the three surgeons with rates greater than

Table 1 Demographic and clinical characteristics of LABS participants with liver biopsies categorized by surgeon biopsy rate

\begin{tabular}{|c|c|c|c|c|}
\hline Characteristic & $\begin{array}{l}\text { All LABS } 1^{\text {st }} \text { surgeries } \\
(n=6382)\end{array}$ & $\begin{array}{l}\text { All biopsies } \\
(n=693)\end{array}$ & $\begin{array}{l}\text { Biopsy rate } \geq 80 \% \\
(n=433)\end{array}$ & $\begin{array}{l}\text { Biopsy rate }<80 \% \\
(n=260)\end{array}$ \\
\hline Age, years (median, Q1 Q3) & $45(36 \sim 54)$ & $45(36 \sim 55)$ & $44(35 \sim 54)$ & $47(37 \sim 56)$ \\
\hline Sex, male $(n, \%)$ & $1347(21 \%)$ & $148(21 \%)$ & $77(18 \%)$ & $71(27 \%)$ \\
\hline Caucasian (n, \%) & $5504(87 \%)$ & $603(88 \%)$ & $393(91 \%)$ & $210(82 \%)$ \\
\hline BMI, kg/m² (median, Q1 Q3) & $46(42 \sim 52)$ & $46(42 \sim 52)$ & $46(42 \sim 50)$ & $49(43 \sim 56)$ \\
\hline Diabetic $(n, \%)$ & $2112(33 \%)$ & $243(35 \%)$ & $132(30 \%)$ & $111(43 \%)$ \\
\hline Abnormal liver appearance $^{\mathrm{a}}(n, \%)$ & $737 / 2419(30 \%)$ & $138 / 283(49 \%)$ & $22 / 32(69 \%)$ & $116 / 251(46 \%)$ \\
\hline ALT, IU/L (median, Q1 Q3) & $26(19 \sim 38)$ & $25(19 \sim 36)$ & $24(18 \sim 34)$ & $27(20 \sim 39)$ \\
\hline AST, IU/L (median, Q1 Q3) & $21(17 \sim 27)$ & $23(19 \sim 28)$ & $22(18 \sim 27)$ & $24(19 \sim 31)$ \\
\hline AP, IU/L (median, Q1 Q3) & $76(63 \sim 93)$ & $71(60 \sim 86)$ & $70(59 \sim 83)$ & $71(61 \sim 88)$ \\
\hline $\begin{array}{l}\text { Fasting glucose, mg/dL } \\
\text { (median, Q1 Q3) }\end{array}$ & $98(88 \sim 118)$ & $100(89 \sim 120)$ & $100(90 \sim 119)$ & $100(88 \sim 128)$ \\
\hline $\begin{array}{l}\text { Fasting insulin }{ }^{\mathrm{a}}, \mathrm{mU} / \mathrm{mL} \\
\text { (median, Q1 Q3) }\end{array}$ & $\begin{array}{l}20(13 \sim 31) \\
(n=2340)\end{array}$ & $\begin{array}{l}22.5(15 \sim 33.5) \\
(n=279)\end{array}$ & $\begin{array}{l}22(14 \sim 28.5) \\
(n=32)\end{array}$ & $\begin{array}{l}23(15 \sim 34) \\
(n=247)\end{array}$ \\
\hline HOMA* (median, Q1 Q3) $^{*}$ & $\begin{array}{l}2.6(1.8 \sim 4.0) \\
(n=2152)\end{array}$ & $\begin{array}{l}2.9(2.0 \sim 4.4) \\
(n=267)\end{array}$ & $\begin{array}{l}2.7(1.9 \sim 3.8) \\
(n=31)\end{array}$ & $\begin{array}{l}2.9(2.0 \sim 4.5) \\
(n=236)\end{array}$ \\
\hline
\end{tabular}

Abbreviations: BMI, body mass index; ALT, alanine aminotransferase; AP, alkaline phosphate; AST, aspartate aminotransferase ${ }^{\mathrm{a}}$ Data collected on LABS-2 participants $(n=2458)$ only. 
Hepatic Pathology among Patients without Known Liver Disease Undergoing Bariatric Surgery Kleiner et al. 101

Table 2 Distribution of histological findings in liver biopsies from LABS participants categorized by surgeon biopsy rate

\begin{tabular}{|c|c|c|c|}
\hline Histological findings & $\begin{array}{l}\text { All biopsies } \\
(n=693)\end{array}$ & $\begin{array}{l}\text { Biopsy rate } \geq 80 \% \\
(n=433)\end{array}$ & $\begin{array}{l}\text { Biopsy rate }<80 \% \\
(n=260)\end{array}$ \\
\hline Biopsy length, mm (median, Q1 Q3) & $12(9 \sim 16)$ & $12(9 \sim 15)$ & $13(10 \sim 18)$ \\
\hline Portal areas (median, Q1 Q3) & $12(8 \sim 17)$ & $11(8 \sim 16)$ & $12(9 \sim 19)$ \\
\hline \multicolumn{4}{|l|}{ Diagnostic classification } \\
\hline Not steatohepatitis & $460(66.4 \%)$ & $317(73.2 \%)$ & $143(55.0 \%)$ \\
\hline Borderline steatohepatitis $^{a}$ & $121(17.4 \%)$ & $70(16.1 \%)$ & $51(19.6 \%)$ \\
\hline Definite steatohepatitis & $112(16.2 \%)$ & $46(10.6 \%)$ & $66(25.4 \%)$ \\
\hline \multicolumn{4}{|l|}{ Steatosis grade } \\
\hline $0 \%$ & $9(1.3 \%)$ & $6(1.4 \%)$ & $3(1.2 \%)$ \\
\hline$<5 \%$ & $137(19.8 \%)$ & $83(19.2 \%)$ & $54(20.8 \%)$ \\
\hline $5-33 \%$ & $323(46.6 \%)$ & $194(44.8 \%)$ & $129(49.6 \%)$ \\
\hline $33-67 \%$ & $148(21.4 \%)$ & $98(22.6 \%)$ & $50(19.2 \%)$ \\
\hline$>67 \%$ & $76(11.0 \%)$ & $52(12.0 \%)$ & $24(9.2 \%)$ \\
\hline \multicolumn{4}{|l|}{ Steatosis location } \\
\hline Zone 3 predominant & $461(67.4 \%)$ & $287(67.2 \%)$ & $174(67.7 \%)$ \\
\hline Zone 1 predominant & $4(0.6 \%)$ & $0(0.0 \%)$ & $4(1.6 \%)$ \\
\hline Panacinar & $123(18.0 \%)$ & $98(23.0 \%)$ & $25(9.7 \%)$ \\
\hline Azonal & $96(14.0 \%)$ & 42 (9.8\%) & $54(21.0 \%)$ \\
\hline Microvesicular steatosis & $43(6.2 \%)$ & $29(6.7 \%)$ & $14(5.4 \%)$ \\
\hline \multicolumn{4}{|l|}{ Lobular inflammation grade } \\
\hline No foci & $48(6.9 \%)$ & $35(8.1 \%)$ & $13(5.0 \%)$ \\
\hline$<2$ foci/20x HPF & $515(74.3 \%)$ & $349(80.6 \%)$ & $166(63.9 \%)$ \\
\hline 2-4 foci/20x HPF & $105(15.2 \%)$ & $41(9.5 \%)$ & $64(24.6 \%)$ \\
\hline$>4$ foci/20x HPF & $25(3.6 \%)$ & $8(1.9 \%)$ & $17(6.5 \%)$ \\
\hline \multicolumn{4}{|l|}{ Portal inflammation } \\
\hline None & $90(13.0 \%)$ & $61(14.1 \%)$ & $29(11.2 \%)$ \\
\hline Mild & 505 (72.9\%) & $332(76.7 \%)$ & $173(66.5 \%)$ \\
\hline More than mild & $98(14.1 \%)$ & $40(9.2 \%)$ & $58(22.3 \%)$ \\
\hline \multicolumn{4}{|l|}{ Ballooning injury } \\
\hline None & 509 (73.5\%) & $336(77.6 \%)$ & $173(66.5 \%)$ \\
\hline Few & 127 (18.3\%) & $73(16.9 \%)$ & $54(20.8 \%)$ \\
\hline Many & $57(8.2 \%)$ & $24(5.5 \%)$ & $33(12.7 \%)$ \\
\hline Acidophil bodies & $129(18.6 \%)$ & $84(19.4 \%)$ & $45(17.4 \%)$ \\
\hline Megamitochondria & $97(14.0 \%)$ & $74(17.1 \%)$ & $23(8.9 \%)$ \\
\hline Mallory-Denk bodies & $38(5.5 \%)$ & $18(4.2 \%)$ & $20(7.7 \%)$ \\
\hline \multicolumn{4}{|l|}{ Fibrosis stage } \\
\hline None & $442(63.9 \%)$ & $317(73.4 \%)$ & $125(48.1 \%)$ \\
\hline Mild perisinusoidal only & $67(9.7 \%)$ & $30(6.9 \%)$ & $37(14.2 \%)$ \\
\hline Moderate perisinuosoidal only & $31(4.5 \%)$ & 17 (3.9\%) & $14(5.4 \%)$ \\
\hline Periportal only & $49(7.1 \%)$ & $31(7.2 \%)$ & $18(6.9 \%)$ \\
\hline Periportal and perisinusoidal & $74(10.7 \%)$ & $29(6.7 \%)$ & $45(17.3 \%)$ \\
\hline Bridging fibrosis & $21(3.0 \%)$ & $6(1.4 \%)$ & $15(5.8 \%)$ \\
\hline Cirrhosis & $8(1.2 \%)$ & $2(0.5 \%)$ & $6(2.3 \%)$ \\
\hline
\end{tabular}


Table 2 (Continued)

\begin{tabular}{|l|l|l|l|}
\hline Histological findings & $\begin{array}{l}\text { All biopsies } \\
(\boldsymbol{n}=\mathbf{6 9 3})\end{array}$ & $\begin{array}{l}\text { Biopsy rate } \geq 80 \% \\
(\boldsymbol{n}=\mathbf{4 3 3})\end{array}$ & $\begin{array}{l}\text { Biopsy rate }<\mathbf{8 0} \\
(\boldsymbol{n}=\mathbf{2 6 0})\end{array}$ \\
\hline \multicolumn{5}{|l|}{ NAFLD activity score (NAS) } & $377(54.4 \%)$ & $249(57.5 \%)$ & $180(69.2 \%)$ \\
\hline 0 to 2 & $221(31.9 \%)$ & $137(31.6 \%)$ & $84(32.3 \%)$ \\
\hline 3 to 4 & $95(13.7 \%)$ & $47(10.9 \%)$ & $48(18.5 \%)$ \\
\hline 5 to 8 & 0.46 & 0.37 & 0.60 \\
\hline Mean Ishak inflammation scores & 1.48 & 1.31 & 1.77 \\
\hline Periportal inflammation & 1.02 & 0.96 & 1.13 \\
\hline Lobular inflammation & 0.06 & 0.06 & 0.07 \\
\hline Portal inflammation & \multicolumn{2}{|l}{} \\
\hline Confluent necrosis &
\end{tabular}

Abbreviations: HPF, high-power field; NAFLD, nonalcoholic fatty liver disease.

${ }^{\mathrm{a}} \mathrm{A}$ single biopsy showed the borderline, zone 1 pattern most often seen in children.

$80 \%$ were compared against the data from surgeons who did not use biopsy as frequently. The biopsies included in the study had a median length of $12 \mathrm{~mm}$ (IQR 9 to $16 \mathrm{~mm}$ ) and a median number of portal areas of 12 (IQR 8 to 17) with a slight tendency toward longer samples with more portal areas in the more selectively obtained biopsies ( - Table 2). Although the differences were not dramatic, biopsied patients of surgeons who performed fewer biopsies were more often male, less often Caucasian, more often diabetic, and had higher BMI, alanine aminotransferase (ALT), and aspartate aminotransferase (AST) (all $p \leq 0.01$ ). As expected, participants sampled more selectively had more inflammation (lobular and portal), more ballooning, more fibrosis, and higher NAS.

The available biopsy data showed a low rate of serious liver disease. Only $4.2 \%$ of biopsies showed bridging fibrosis or cirrhosis, and only $13.7 \%$ had a NAS of 5 or greater. With respect to diagnostic categorization, only $16.2 \%$ had definite steatohepatitis, although an additional $17.4 \%$ had borderline steatohepatitis, including one participant who had the zone 1 borderline pattern associated with fatty liver disease in young children. ${ }^{11}$ Histological analyses also included scoring inflammation and fibrosis using the Ishak scoring system (-Table 2). More than $40 \%$ of the biopsies had evidence of interface hepatitis although in only $3.2 \%$ of cases did this involve more than half of the portal areas (Ishak periportal inflammation score $>1$ ). Moderate portal inflammation (Ishak portal inflammation score $>1$ ) was seen in $15.7 \%$. Larger foci of confluent lobular inflammation or severe perivenular inflammation were found in only $4.6 \%$ (Ishak confluent necrosis $\geq 1$ ).

The histological characteristics of the biopsies as defined by ALT values are summarized in -Table 3 and -Fig. 1. The histological changes are generally milder in patients with ALTs $<$ ULN under the stricter Prati criteria than under the LABS criteria, but the differences are not great. Even using the lower Prati values for the ULN for ALT, 3.7\% of biopsies have NAS $\geq 5$, $1.6 \%$ have a fibrosis stage $\geq 3$ (bridging or cirrhosis), and $7.9 \%$ have definite steatohepatitis. Other important histological finding of hepatocyte injury were also noted among those with "normal" ALT, including 10.1\% with acidophil bodies, $13.2 \%$ with megamitochondria, $4.2 \%$ with microvesicular steatosis, and $1.1 \%$ with Mallory-Denk bodies. While an absolute level of ALT is a poor test to exclude disease, participants with ALT $\geq 35$ at the time of bariatric surgery had a high rate of significant disease, with $35.5 \%$ having NAS $\geq 5,10.7 \%$ having fibrosis stage $\geq 3$, and $35.5 \%$ having definite steatohepatitis.

To estimate the proportion of participants with significant disease in the unbiopsied LABS-2 sample for whom ALT data were available $(n=1970)$, we developed logistic regression models based on data from 264 LABS-2 participants with data for age, sex, ALT and biopsy results to predict the probability of having a significant fibrosis stage (bridging fibrosis or cirrhosis) (area under ROC; i.e., c-statistic $=0.65$ ) or of having the diagnosis of borderline or definite steatohepatitis (area under ROC; i.e., c-statistic $=0.77$ ), adjusting for age, sex and ALT ( - Table 4). It is important to note that the biopsied LABS2 population used for this analysis had worse disease than the LABS-1/LABS-2 population described in - Table 2. The fibrosis stage model predicted that 117 (95\% CI: $67-199)$ or $5.9 \%$ (95\% CI: $3.4-10.1 \%$ ) of these 1,970 unbiopsied LABS-2 participants were likely to have bridging fibrosis or cirrhosis. The proportion of biopsied participants with significant fibrosis stage was about the same as the modeled results from the unbiopsied cohorts ( $7.2 \%$ vs. $5.9 \%$ ), that is the $95 \%$ Cls overlapped (4.1-10.3\% vs. 3.4-10.1\%). With respect to diagnosis, a separate model predicted that 898 (95\% Cl: $751-1049$ ) or $45.6 \%$ (95\%CI: 38.1-53.2\%) of the 1970 unbiopsied participants were likely to have borderline or definite steatohepatitis. Based on these models it is estimated that $86.0 \%$ of participants with advanced fibrosis and $88.1 \%$ of those with borderline or definite steatohepatitis in our cohort were not biopsied and went undiagnosed.

\section{Discussion}

A recent PubMed search identified 36 reports describing the hepatic histology observed in $\sim 7,000$ liver biopsies obtained during bariatric surgery over the past 25 years. They 
Table 3 Distribution of selected histological findings in liver biopsies from LABS participants according to ALT level

\begin{tabular}{|c|c|c|c|}
\hline Histological findings & $\begin{array}{l}\mathrm{ALT} \geq 35 \mathrm{IU} / \mathrm{L} \\
(n=169)\end{array}$ & $\begin{array}{l}\text { ALT }<35 \mathrm{IU} / \mathrm{L} \\
(n=466)^{\mathrm{a}}\end{array}$ & $\begin{array}{l}\text { ALT }<30 \mathrm{IU} / \mathrm{L} \text { for men or } \\
\text { ALT }<19 \mathrm{IU} / \mathrm{L} \text { for women } \\
(n=190)^{\mathrm{b}}\end{array}$ \\
\hline \multicolumn{4}{|l|}{ Diagnostic classification } \\
\hline Not steatohepatitis & 67 (39.6\%) & $354(76.0 \%)$ & $155(81.6 \%)$ \\
\hline Borderline steatohepatitis & $42(24.9 \%)$ & $68(14.6 \%)$ & $20(10.5 \%)$ \\
\hline Definite steatohepatitis & 60 (35.5\%) & $44(9.4 \%)$ & $15(7.9 \%)$ \\
\hline \multicolumn{4}{|l|}{ Steatosis grade } \\
\hline $0 \%$ & $1(0.6 \%)$ & $8(1.7 \%)$ & $5(2.6 \%)$ \\
\hline$<5 \%$ & 7 (4.1\%) & $116(24.9 \%)$ & $62(32.6 \%)$ \\
\hline $5-33 \%$ & 66 (39.1\%) & $232(49.8 \%)$ & $98(51.6 \%)$ \\
\hline $33-67 \%$ & $50(29.6 \%)$ & $84(18.0 \%)$ & $20(10.5 \%)$ \\
\hline$>67 \%$ & $45(26.6 \%)$ & $26(5.6 \%)$ & $5(2.6 \%)$ \\
\hline \multicolumn{4}{|l|}{ Lobular inflammation grade } \\
\hline No foci & $2(1.2 \%)$ & $43(9.2 \%)$ & $19(10.0 \%)$ \\
\hline$<2$ foci/20x HPF & $96(56.8 \%)$ & $370(79.4 \%)$ & $153(80.5 \%)$ \\
\hline 2-4 foci/20x HPF & $53(31.4 \%)$ & 47 (10.1\%) & $16(8.4 \%)$ \\
\hline$>4$ foci/20x HPF & $18(10.7 \%)$ & $6(1.3 \%)$ & $2(1.1 \%)$ \\
\hline \multicolumn{4}{|l|}{ Portal inflammation } \\
\hline None & $11(6.5 \%)$ & $70(15.0 \%)$ & $29(15.3 \%)$ \\
\hline Mild & $115(68.1 \%)$ & $351(75.3 \%)$ & $142(74.7 \%)$ \\
\hline More than mild & $43(25.4 \%)$ & $45(9.7 \%)$ & $19(10.0 \%)$ \\
\hline \multicolumn{4}{|l|}{ Ballooning injury } \\
\hline None & 94 (55.6\%) & $370(79.4 \%)$ & $160(84.2 \%)$ \\
\hline Few & $40(23.7 \%)$ & $79(17.0 \%)$ & $27(14.2 \%)$ \\
\hline Many & $35(20.7 \%)$ & $17(3.6 \%)$ & $3(1.6 \%)$ \\
\hline \multicolumn{4}{|l|}{ Fibrosis stage } \\
\hline None & $59(34.9 \%)$ & $345(74.2 \%)$ & $138(72.6 \%)$ \\
\hline Mild perisinusoidal only & $19(11.2 \%)$ & $45(9.7 \%)$ & $19(10.0 \%)$ \\
\hline Moderate perisinuosoidal only & $18(10.7 \%)$ & $12(2.6 \%)$ & $2(1.1 \%)$ \\
\hline Periportal only & $11(6.5 \%)$ & $34(7.3 \%)$ & $21(11.1 \%)$ \\
\hline Periportal and perisinusoidal & $44(26.0 \%)$ & $20(4.3 \%)$ & $7(3.7 \%)$ \\
\hline Bridging fibrosis & $14(8.3 \%)$ & $5(1.1 \%)$ & $1(0.5 \%)$ \\
\hline Cirrhosis & $4(2.4 \%)$ & $4(0.9 \%)$ & $2(1.1 \%)$ \\
\hline \multicolumn{4}{|l|}{ NAFLD activity score (NAS) } \\
\hline 0 to 2 & $44(26.0 \%)$ & $300(64.4 \%)$ & $141(74.2 \%)$ \\
\hline 3 to 4 & 65 (38.5\%) & $136(29.2 \%)$ & $42(22.1 \%)$ \\
\hline 5 to 8 & $60(35.5 \%)$ & $30(6.4 \%)$ & $7(3.7 \%)$ \\
\hline \multicolumn{4}{|l|}{ Mean Ishak Inflammation Scores } \\
\hline Periportal Inflammation & 0.63 & 0.39 & 0.37 \\
\hline Lobular Inflammation & 2.06 & 1.29 & 1.22 \\
\hline Portal Inflammation & 1.21 & 0.94 & 0.93 \\
\hline Confluent Necrosis & 0.14 & 0.03 & 0.04 \\
\hline
\end{tabular}

Abbreviations: ALT, alanine aminotransferase HPF, high-power field.

aStandard LABS upper limit of normal (ULN).

bPrati criteria for ULN. 


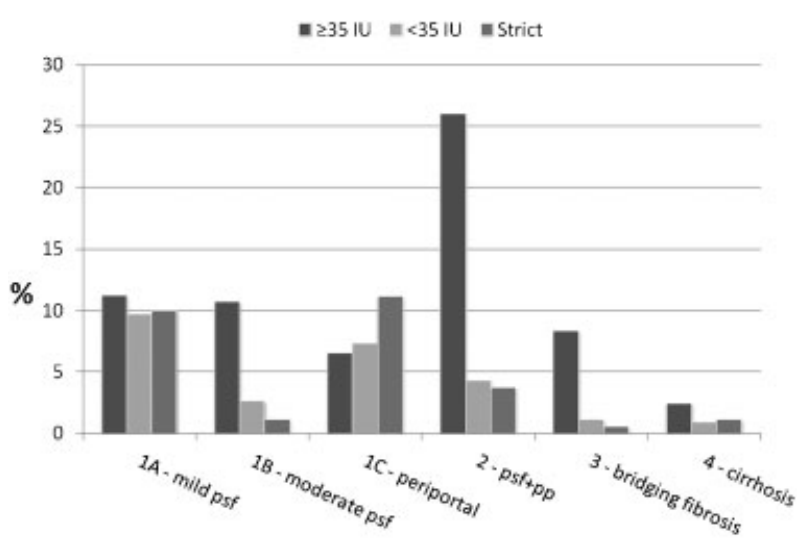

A

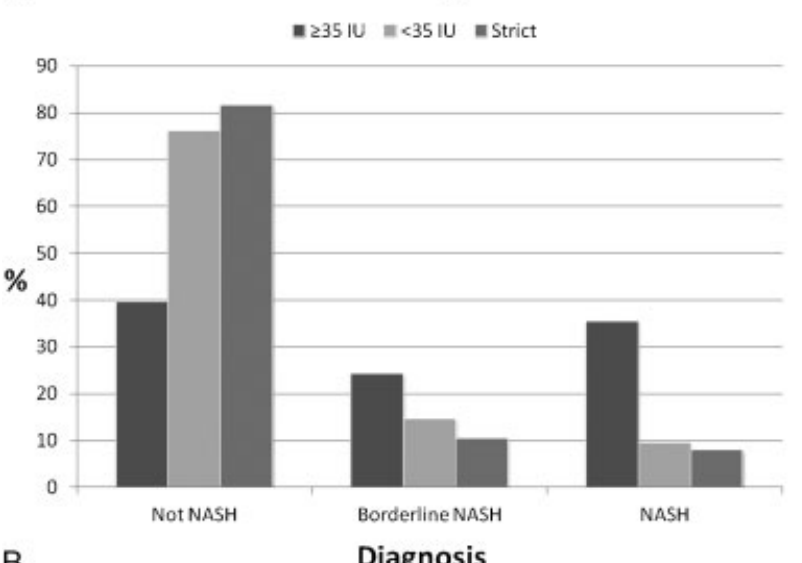

B Diagnosis

Fig. 1 Distribution of fibrosis and diagnostic categories in cohorts of participants defined by alanine aminotransferase (ALT) levels. The "strict" criteria for ALT are the criteria for the upper limit of normal defined by Prati et al, < $30 \mathrm{IU} / \mathrm{L}$ for men and $<19 \mathrm{IU} / \mathrm{L}$ for women. ${ }^{29}$ (A) Distribution of fibrosis by ALT level (the category of "no fibrosis" omitted for clarity). psf = perisinusoidal fibrois, $\mathrm{pp}=$ periportal fibrosis (see - Table 3). (B) Distribution of diagnostic category by ALT level. described series of varying size, of which the largest, at 679 and 689 biopsies, respectively, ${ }^{34,35}$ were similar in size to the current cohort. Most of these studies observed the entire spectrum of NAFLD, although the relative proportions of the various elements varied appreciably, in part because of changes in diagnostic criteria over time, different pathologists, and differences in the circumstances under which biopsies were collected. In two series that examined histology in the light of clinical test results, cases with significant hepatic injury including cirrhosis were found not infrequently in the presence of normal serum aminotransferases, ${ }^{36,37}$ although in one series the ULN for ALT was considered to be 72 $\mathrm{IU} / \mathrm{L}{ }^{37}$ Thus, although it is clearly common, the overall severity and long term implications of liver disease in bariatric surgical patients remain unclear, as does the appropriate approach to screen them for liver disease. Given the growing increase in morbidity and mortality associated with the increased incidence of end-stage obesity-associated liver disease and the growing financial burden of liver transplantation in the population, these two unanswered questions are becoming increasingly critical.

Reflecting differences of opinion among LABS surgeons as to the importance of routine liver biopsy during bariatric surgery, liver biopsies were not a required part of any LABS study. Instead, liver biopsies were performed as part of the standard of care at each institution, according to the individual practices of the participating surgeons. Our data show that there is wide institutional variation in biopsy rates between surgeons, with some surgeons biopsying all patients and others biopsying none. We found small but significant differences in the frequency with which serious histologic abnormalities were detected by surgeons who routinely biopsied most cases and those who performed liver biopsies more selectively, if at all. We also demonstrated that an appreciable minority of participants with "normal" ALTs both by the LABS consensus criteria and by the stricter criteria of Prati et $\mathrm{al}^{29}$ nevertheless have evidence of significant liver

Table 4 Observed and predicted significant diseases (fibrosis stage and steatohepatitis) from LABS-2 participants

\begin{tabular}{|c|c|c|c|}
\hline Disease & $\begin{array}{l}\text { Observed from } \\
\text { Biopsied LABS-2 sample } \\
\left(n=264^{b}\right)\end{array}$ & $\begin{array}{l}\text { Predicted }^{\mathrm{a}} \text { from } \\
\text { Unbiopsied LABS-2 sample } \\
\left(n=1970^{\mathrm{b}}\right)\end{array}$ & $\begin{array}{l}\text { LABS-2 sample } \\
\left(n=2234^{\text {b }}\right)\end{array}$ \\
\hline \multicolumn{4}{|l|}{ Fibrosis stage } \\
\hline Significant ${ }^{\mathrm{c}}$ & $\begin{array}{l}19 \text { (7.2\%) } \\
95 \% \text { Cl: (4.1\% 10.3\%) }\end{array}$ & $\begin{array}{l}117 \text { (5.9\%) } \\
95 \% \text { Cl: } 3.4 \% \sim 10.1 \%\end{array}$ & 136 \\
\hline Not significant & 245 (92.8\%) & 1853(94.1\%) & 2143 \\
\hline \multicolumn{4}{|l|}{ Steatohepatitis } \\
\hline Significant ${ }^{\mathrm{d}}$ & $\begin{array}{l}\text { 121(45.8\%) } \\
95 \% \text { Cl: } 39.7 \% \sim 52.1 \%\end{array}$ & $\begin{array}{l}898 \text { (45.6\%) } \\
95 \% \text { Cl: } 38.1 \% \sim 53.2 \%\end{array}$ & 1019 \\
\hline Not significant & 143 (54.2\%) & 1072 (54.4\%) & 1260 \\
\hline
\end{tabular}

Abbreviations: $\mathrm{Cl}$, confidence interval.

aLogistic regression model, adjusting for age, sex, and alanine aminotransferase (ALT).

barticipants with missing values of biopsy data and ALT were not included.

'Bridging or cirrhosis fibrosis stage.

${ }^{\mathrm{d} B o r d e r l i n e ~ o r ~ d e f i n i t e ~ s t e a t o h e p a t i t i s . ~}$ 
disease. Although our surgeons who biopsied selectively identified a higher percentage of cases of advanced liver fibrosis (8.1\% vs. $1.8 \%)$ and definite steatohepatitis $(25.4 \%$ vs. $10.6 \%$, our logistic regression models estimated that between 67 and 199 participants in our cohorts had undiagnosed advanced fibrosis and between 751 and 1049 had undiagnosed steatohepatitis, conditions that can progress to end-stage liver disease in a not insignificant proportion of affected patients. ${ }^{11,16,38}$ By contrast, the evidence that this progression will be prevented by bariatric surgery is less than clear. Thus, while there may be valid reasons for not biopsying all morbidly obese bariatric surgery patients, these results suggest that not biopsying everyone is also a suboptimal strategy.

Several studies have demonstrated that the ability of surgeons to identify significant liver disease during surgery is limited and unreliable. ${ }^{39,40}$ It is often assumed that a "normal" ALT value is a surrogate marker for the absence of significant liver disease. However, unlike many measurements used in routine clinical chemistry, ALT normal ranges are not determined from validated standards. Instead, they are determined using varying procedures and types of instrumentation on samples collected from local reference populations, ${ }^{41}$ in which such issues as diet and incidence of obesity may appreciably impact the results. Consequently, the ULN for ALT varies almost twofold among major laboratories. ${ }^{41}$ Much of the variability is believed to derive from differences in the local populations sampled to define the ULN. Accordingly, at the start of LABS our protocol defined the ULN for ALT to be $35 \mathrm{IU} / \mathrm{L}$ to standardize the evaluation and interpretation of ALT values reported from 11 sites around the country. Subsequently, others have suggested lower values for the ULN based on measurements in patients without any evidence of liver disease. In particular, Prati et al recommended ULN of $<30$ $\mathrm{IU} / \mathrm{L}$ in men and $<19 \mathrm{IU} / \mathrm{L}$ in women. ${ }^{29}$ Accordingly, in this study, we used the laboratory data and standard of care biopsies obtained as part of LABS to see if the stricter Prati limits for the normal range of ALT would effectively exclude serious liver disease in the LABS samples. However, a significant minority of participants with "normal" ALT by the Prati criteria nevertheless had evidence of serious liver disease, including $18.4 \%$ with some degree of steatohepatitis and $5.3 \%$ with fibrosis of stage 2 or worse. These data indicate that physicians and surgeons evaluating patients for bariatric surgery cannot assume that a normal range ALT value indicates an absence of significant liver disease.

Because the LABS study overall was not designed to assess liver disease across the bariatric surgery population, some limitations should be noted. That only standard-of-care biopsies were obtained for this study resulted in nonrandom assessment of $10.9 \%$ of LABS participants. Testing to exclude other liver disease (particularly chronic hepatitis C) was not rigorously performed, so that our sample likely includes 2 to $3 \%$ of participants with viral hepatitis. These may account for some of the cases with more severe portal and periportal inflammation. Nevertheless, the proportion of participants with steatohepatitis, steatosis and advanced fibrosis is well within the range of findings by others with more systematic sampling. ${ }^{34,35}$ Moreover, biopsies performed selectively did not show dramatically different histological findings from those obtained systematically. The main analyses performed in this study, evaluation of the histologic spectrum observed in participants with normal ALT, should not be as affected by the way in which our participant population was sampled. The Ishak scoring system, included in this study for the sake of completeness, is most often used in the evaluation of chronic hepatitis. It does not include evaluation of perisinusoidal fibrosis, an important feature of NASH, but does provide a more detailed grading of inflammation and interface hepatitis than other systems routinely employed. Although of value for investigative purposes, it is unclear if the added information it provides justifies its inclusion in routine clinical diagnostic studies. Finally, the relatively simple models employed to explore the potential for missed pathology in the nonbiopsied patients of the LABS- 2 cohort were not validated in an independent dataset, and their prediction performance characteristics were imperfect (c-statistics 0.65-0.77). Although this limits to some degree the precision of our model-based predictions, the computed CIs nevertheless strongly suggest that considerable significant pathology is being missed by current sampling procedures.

In summary, the bariatric surgery population is at appreciable risk for significant and potentially fatal liver disease even in many patients who present with normal ALT levels. Our data demonstrate that a normal ALT, even if strictly defined, is not a reliable surrogate marker for a healthy liver. Development of an accurate noninvasive method for assessing the state of the liver preoperatively should have a high priority, as should well-designed studies to follow the evolution of biopsy proven liver disease after bariatric surgery. Adequate data on this key issue do not yet exist. ${ }^{42}$ However, while currently available noninvasive tests are inadequate to reliably detect significant liver disease in the LABS population, liver biopsy can detect it, and in the setting of a laparoscopic bariatric procedure, can do it both safely and inexpensively. It has been argued that there is no need to make an early diagnosis or follow such patients because there is currently no agreed-upon, effective medical therapy for NASH. This lack of an agreed-upon medical treatment approach is conceded even in the NASH treatment guidelines published jointly by the American Gastroenterological Association, American Association for the Study of Liver Diseases, and the American College of Gastroenterology, ${ }^{38}$ which review the multiple options currently available to treating physicians. However, the analogy to hepatitis $C$ is instructive. It became apparent recently that hepatitis $C$ was suddenly, after a quarter century of therapeutic frustration, a medically curable disease. The euphoria was brief since, due to a failure of screening programs worldwide, 75 to $90 \%$ of hepatitis C virus-infected patients are asymptomatic, unaware of their disease, and do not present for treatment until it is far advanced. As with hepatitis C, there will, eventually, be effective medical therapies for NASH. Afflicted patients will surely be better off knowing they have it and being followed by physicians trained and ready to offer them new, effective treatment options as soon as they are available, rather than 
waiting to be treated only after serious symptoms announce the diagnosis.

Liver biopsy during bariatric surgery offers a key opportunity to diagnose an important and currently underdiagnosed illness, and to offer medical follow-up to affected patients. Liver biopsy should be considered routinely during bariatric surgery.

\section{Acknowledgments}

LABS was funded by a cooperative agreement (Grant DCC U01 DK066557) by the National Institute of Diabetes and Digestive and Kidney Diseases; Grant U01-DK66667 to Columbia-Presbyterian Hospital, New York, NY in collaboration with Grant UL1-RR024996 to Cornell University Medical Center, Clinical and Translational Research Center (CTRC), Ithaca, NY; Grant U01-DK66568 to the University of Washington, Seattle, WA in collaboration with Grant M01RR-00037 from CTRC; Grant U01-DK66471 to the Neuropsychiatric Research Institute, Fargo, ND; Grant U01-DK66526 to East Carolina University, Greenville, NC; Grant U01-DK66585 to the University of Pittsburgh Medical Center, Pittsburgh, PA in collaboration with Grant UL1-RR024153 from CTRC; and Grant U01-DK66555 to Oregon Health and Science University, Portland, OR. This liver biopsy research project was supported in part by the Intramural Research Program of the National Cancer Institute, $\mathrm{NIH}$.

\section{References}

1 Flegal KM, Carroll MD, Kit BK, Ogden CL. Prevalence of obesity and trends in the distribution of body mass index among US adults, 1999-2010. JAMA 2012;307(5):491-497

2 Flegal KM, Carroll MD, Ogden CL, Johnson CL. Prevalence and trends in obesity among US adults, 1999-2000. JAMA 2002; 288(14):1723-1727

3 Ogden CL, Carroll MD, Curtin LR, McDowell MA, Tabak CJ, Flegal KM. Prevalence of overweight and obesity in the United States, 1999-2004. JAMA 2006;295(13):1549-1555

4 Ogden CL, Yanovski SZ, Carroll MD, Flegal KM. The epidemiology of obesity. Gastroenterology 2007;132(6):2087-2102

5 Clark JM. The epidemiology of nonalcoholic fatty liver disease in adults. J Clin Gastroenterol 2006;40(Suppl 1):S5-S10

6 Younossi ZM, Stepanova M, Afendy M, et al. Changes in the prevalence of the most common causes of chronic liver diseases in the United States from 1988 to 2008. Clin Gastroenterol Hepatol 2011;9(6):524-530, e1, quiz e60

7 Liu CJ. Prevalence and risk factors for non-alcoholic fatty liver disease in Asian people who are not obese. J Gastroenterol Hepatol 2012;27(10):1555-1560

8 Bray GA. Medical consequences of obesity. J Clin Endocrinol Metab 2004;89(6):2583-2589

9 Brunt EM, Kleiner DE, Wilson LA, et al; NASH Clinical Research NetworkA list of members of the Nonalcoholic Steatohepatitis Clinical Research Network can be found in the Appendix. Portal chronic inflammation in nonalcoholic fatty liver disease (NAFLD): a histologic marker of advanced NAFLD-Clinicopathologic correlations from the nonalcoholic steatohepatitis clinical research network. Hepatology 2009;49(3):809-820

10 Brunt EM, Tiniakos DG. Pathological features of NASH. Front Biosci 2005;10:1475-1484
11 Kleiner DE, Brunt EM. Nonalcoholic fatty liver disease: pathologic patterns and biopsy evaluation in clinical research. Semin Liver Dis 2012;32(1):3-13

12 Kleiner DE, Brunt EM, Van Natta M, et al; Nonalcoholic Steatohepatitis Clinical Research Network. Design and validation of a histological scoring system for nonalcoholic fatty liver disease. Hepatology 2005;41(6):1313-1321

13 Zelman S. The liver in obesity. AMA Arch Intern Med 1952;90(2): 141-156

14 Thaler H. Morphologische befunde bei chronischer alkoholintoxikation [Morphologic findings in chronic alcohol intoxication]. In: Gerok W, Sickinger K, Hennkeuser HH, eds. Alcohol and the Liver. Stuttgart, Germany: Schattauer Verlag; 1971:257-265

15 Ludwig J, Viggiano TR, McGill DB, Oh BJ. Nonalcoholic steatohepatitis: Mayo Clinic experiences with a hitherto unnamed disease. Mayo Clin Proc 1980;55(7):434-438

16 Powell EE, Cooksley WG, Hanson R, Searle J, Halliday JW, Powell LW. The natural history of nonalcoholic steatohepatitis: a followup study of forty-two patients for up to 21 years. Hepatology 1990; 11(1):74-80

17 Viral Hepatitis Surveillance - United States. 2010. Centers for Disease Control; 2012.

18 Centers for Disease Control. Reported cases of acute viral hepatitis, by type and year, United States, 1966-2003. Atlanta, GA: Centers for Disease Control; 2012

19 Aghemo A, De Francesco R. New horizons in hepatitis C antiviral therapy with direct-acting antivirals. Hepatology 2013;58(1): 428-438

20 Lawitz E, Poordad F, Kowdley KV, et al. A phase 2a trial of 12-week interferon-free therapy with two direct-acting antivirals (ABT$450 / r, A B T-072$ ) and ribavirin in IL28B C/C patients with chronic hepatitis C genotype 1. J Hepatol 2013;59(1):18-23

21 Pawlotsky JM. Treatment of chronic hepatitis C: current and future. Curr Top Microbiol Immunol 2013;369:321-342

22 Vachon ML, Dieterich DT. The era of direct-acting antivirals has begun: the beginning of the end for HCV? Semin Liver Dis 2011; 31(4):399-409

23 Burke A, Lucey MR. Non-alcoholic fatty liver disease, non-alcoholic steatohepatitis and orthotopic liver transplantation. Am J Transplant 2004;4(5):686-693

24 Charlton MR, Burns JM, Pedersen RA, Watt KD, Heimbach JK, Dierkhising RA. Frequency and outcomes of liver transplantation for nonalcoholic steatohepatitis in the United States. Gastroenterology 2011;141(4):1249-1253

25 Merion RM. Current status and future of liver transplantation. Semin Liver Dis 2010;30(4):411-421

26 Sanyal AJ, Chalasani N, Kowdley KV, et al; NASH CRN. Pioglitazone, vitamin E, or placebo for nonalcoholic steatohepatitis. N Engl J Med 2010;362(18):1675-1685

27 Speliotes EK, Yerges-Armstrong LM, Wu J, et al; NASH CRN; GIANT Consortium; MAGIC Investigators; GOLD Consortium. Genomewide association analysis identifies variants associated with nonalcoholic fatty liver disease that have distinct effects on metabolic traits. PLoS Genet 2011;7(3):e1001324

28 Belle S, Consortium L; LABS Consortium. The NIDDK Bariatric Surgery clinical Research Consortium (LABS). Surg Obes Relat Dis 2005;1(2):145-147

29 Prati D, Taioli E, Zanella A, et al. Updated definitions of healthy ranges for serum alanine aminotransferase levels. Ann Intern Med 2002;137(1):1-10

30 Belle SH, Berk PD, Courcoulas AP, et al; Longitudinal Assessment of Bariatric Surgery Consortium Writing Group. Safety and efficacy of bariatric surgery: Longitudinal Assessment of Bariatric Surgery. Surg Obes Relat Dis 2007;3(2):116-126

31 Flum DR, Belle SH, King WC, et al; Longitudinal Assessment of Bariatric Surgery (LABS) Consortium. Perioperative safety in the longitudinal assessment of bariatric surgery. N Engl J Med 2009; 361(5):445-454 
32 Belle SH, Berk PD, Chapman WH, et al; LABS Consortium. Baseline characteristics of participants in the Longitudinal Assessment of Bariatric Surgery-2 (LABS-2) study. Surg Obes Relat Dis 2013;9(6): 926-935 [Epub ahead of print]

33 Ishak K, Baptista A, Bianchi L, et al. Histological grading and staging of chronic hepatitis. J Hepatol 1995;22(6):696-699

34 Bedossa P, Poitou C, Veyrie N, et al. Histopathological algorithm and scoring system for evaluation of liver lesions in morbidly obese patients. Hepatology 2012;56(5):1751-1759

35 Kral JG, Thung SN, Biron S, et al. Effects of surgical treatment of the metabolic syndrome on liver fibrosis and cirrhosis. Surgery 2004; 135(1):48-58

36 Kunde SS, Lazenby AJ, Clements RH, Abrams GA. Spectrum of NAFLD and diagnostic implications of the proposed new normal range for serum ALT in obese women. Hepatology 2005;42(3): 650-656

37 Mofrad P, Contos MJ, Haque M, et al. Clinical and histologic spectrum of nonalcoholic fatty liver disease associated with normal ALT values. Hepatology 2003;37(6):1286-1292
38 Chalasani N, Younossi Z, Lavine JE, et al. The diagnosis and management of non-alcoholic fatty liver disease: practice Guideline by the American Association for the Study of Liver Diseases, American College of Gastroenterology, and the American Gastroenterological Association. Hepatology 2012;55(6):2005-2023

39 Dolce CJ, Russo M, Keller JE, et al. Does liver appearance predict histopathologic findings: prospective analysis of routine liver biopsies during bariatric surgery. Surg Obes Relat Dis 2009;5(3):323-328

40 Teixeira AR, Bellodi-Privato M, Carvalheira JB, Pilla VF, Pareja JC, D'Albuquerque LA. The incapacity of the surgeon to identify NASH in bariatric surgery makes biopsy mandatory. Obes Surg 2009; 19(12):1678-1684

41 Neuschwander-Tetri BA, Unalp A, Creer MH; Nonalcoholic Steatohepatitis Clinical Research Network. Influence of local reference populations on upper limits of normal for serum alanine aminotransferase levels. Arch Intern Med 2008;168(6):663-666

42 Verna EC, Berk PD. Role of fatty acids in the pathogenesis of obesity and fatty liver: impact of bariatric surgery. Semin Liver Dis 2008; 28(4):407-426 